\title{
Gegen die Zwänge des Marktes: Konturen eines demokratischeren Mediensystems
}

\author{
Florian Zollmann
}

Keywords: Medienreformen, Krise des Journalismus, Demokratie, Markt, Kapitalismus

\section{Abstract}

Finanzeinbrüche, Redaktionsverschlankungen, Zusammenschlüsse: Auf lokaler Ebene gibt es kaum noch eigenständige Berichterstattung und der überregionale Journalismus hat seine pluralistische Vielfalt eingebüßt. Der Rundfunk ist durch eine zunehmende Boulevardisierung und Entpolitisierung gekennzeichnet. Das hat Folgen für die Demokratie: Von der Gesellschaft gewünschte Funktionen der Medien, wie Abbildung unterschiedlicher Meinungen, Wahrheitssuche oder objektive Informationsvermittlung, können kaum noch gewährleistet werden. Die Nachrichtenbranche befindet sich in einer Krise, deren Ursache in den Institutionen des kapitalistischen Marktsystems zu finden ist. Medienreformen sind daher dringend nötig. Ziel dieses Beitrages ist - neben einer kritischen Bestandsaufnahme des Journalismus - Visionen und Strategien für Medienreformen herauszuarbeiten, die von einer sozialen Bewegung eingeleitet und mit Blick auf die deutsche Medienlandschaft realisiert werden können. Darunter fallen folgende Aspekte: Konzentrationskontrolle, unabhängige Aufsicht und Überwachung journalistischer Standards, Demokratisierung der Presse- und Rundfunkräte, Aufbau und Finanzierung unabhängiger Nachrichtenmedien, öffentliche Kontrolle und journalistische Selbstverwaltung. Medienreform wird außerdem als Mediengerechtigkeit verstanden, die durch Prinzipien wie Egalitarismus und Demokratie sowie finanzieller und ökologischer Nachhaltigkeit charakterisiert sein sollte. 
Der Autor bedankt sich bei Nils S. Borchers, Kerem Schamberger und Werner Eiermann für konstruktive Kritik und wertvolles Feedback zu einer früheren Version dieses Textes.

Florian Zollmann: Gegen die Zwänge des Marktes: Konturen eines demokratischeren Mediensystems. In: Nils S. Borchers, Selma Güney, Uwe Krüger und Kerem Schamberger (Hrsg.): Transformation der Medien - Medien der Transformation. Verhandlungen des Netzwerks Kritische Kommunikationswissenschaft. Frankfurt am Main: Westend 2021. DOI: https://doi. org/10.53291/BQPE5410.

Dr. Florian Zollmann I Newcastle University, UK I florian.zollmann@ newcastle.ac.uk

\section{Einführung}

Natalie Fenton et al. $(2020,84)$ schreiben in ihrem Manifest für eine Medienreform, die Aufgabe von emanzipatorischen Sozialwissenschaften sei es, auf der Basis einer systematischen und kritischen Untersuchung des gegenwärtigen Gesellschaftszustands Alternativen zu entwickeln. ${ }^{1}$ Ihnen geht es darum, zunächst die Handlungslogiken von existierenden Medienorganisationen sowie die Funktionen der wesentlichen Institutionen innerhalb der politischen Ökonomie der Medienindustrie zu erfassen und ihre Defizite herauszuarbeiten (ebd.). Aufbauend auf dieser Kritik des Status quo, welche die Legitimität von Medienmacht in Frage stellt, können dann die Strukturen eines neuen Mediensystems und Strategien der Medienreform entwickelt werden (ebd.). In diesem Sinn beleuchtet der anschließende Abschnitt zunächst die Ursachen der Krise des Journalismus in Deutschland. Darauf folgt eine kritische Analyse der Institutionen der kapitalistischen Medienindustrie. Der Hauptteil stellt im Anschluss kurz-, mittel-, und langfristig realisierbare Visionen und Strategien für Medienreformen vor. Abschließend werden die Konturen eines demokratischeren Mediensystems, das vielfältigen Akteur*innen der Zivilgesellschaft Zugang zu Medien gewährt und von der Gesellschaft gewünschte Funktionen der Nachrichtenmedien wie Meinungsvielfalt, Wahrheitssuche oder objektive Politik- und Informationsvermittlung leisten kann, aufgezeigt.

1 Fenton et al. $(2020,84)$ beziehen sich hier insbesondere auf Erik Olin Wright. 


\section{Die Krise des Journalismus}

In seiner Festrede zur Verleihung der Otto-Brenner-Preise für kritischen Journalismus am 19. November 2018 in Berlin warnte Armin Wolf, stellvertretender ORF-Chefredakteur, vor einer »Krise des Journalismus«, die ein Symptom darstelle »für etwas noch viel Bedrohlicheres, für eine Krise der Demokratie « (2019). Er verwies auf das traditionelle Geschäftsmodell des Journalismus, das darauf beruht, Publikum an die Werbeindustrie zu verkaufen sowie auf einen zunehmenden Einfluss der Politik auf die Pressefreiheit. Dieses System Journalismus sei durch die Digitalisierung und durch Einschnitte in die Pressefreiheit in seinen Grundfesten erschüttert worden (ebd.).

Das Internet hat den bereits durch Kürzungen gebeutelten Zeitungsunternehmen die Existenzgrundlage weiter entzogen. Denn die werbetreibende Industrie bucht Werberaum und -zeit immer weniger in Nachrichtenmedien, sondern auf digitalen Plattformen wie Facebook und Google (Barthel 2018). Um ein Beispiel zu nennen: 1999 verdienten deutsche Tageszeitungen netto noch etwa sechs Milliarden Euro an Werbeschaltungen, 2019 waren es nur noch knapp über zwei Milliarden Euro (Röper 2020, 333). Solche Einnahmeverluste erhöhen den wirtschaftlichen Druck auf Zeitungsunternehmen und müssen mittels Verkaufserlösen, Mehrfachverwertung von Inhalten und Redaktionsabbau kompensiert werden. »Die stetig sinkenden Gesamteinnahmen führen seit Jahren zu Einsparungen in allen Bereichen«, schreibt Horst Röper (ebd.) im aktuellen Bericht zur Konzentration der Tagespresse. Demnach gibt es eine voranschreitende »Ausdünnung des lokalen Zeitungsangebots«, die zur Folge hat, dass »die Pressevielfalt mehr und mehr« eingeschränkt wird (ebd., 331). Auf überregionaler Ebene gestaltet sich die Zeitungsberichterstattung des Weiteren »immer uniformer, «enn Verlage verzichten auf eigenständige Hauptredaktionen zugunsten von Gemeinschaftsredaktionen, Mantelproduktionen oder Zulieferungen anderer Verlagshäuser (ebd., 332-333).

Das deutsche Rundfunksystem ist von den ökonomischen Folgen der Digitalisierung bisher weniger stark betroffen als die Zeitungsverlage: Die jährlichen Nettowerbeumsätze, die hauptsächlich dem privaten Fernsehen zugutekommen, liegen seit 2007 relativ gleichbleibend bei etwa vier Milliarden Euro (Ferschli, Grabner und Theine 2019, 10); die Finanzierung der Öffentlich-Rechtlichen ist durch den Rundfunkstaatsvertrag gewährleistet. Aber auch der Rundfunk ist dem ökonomischen Diktat der Wirtschaft unterworfen, mit Folgen für die Programmstruk- 
tur und Inhalte. Zahlreiche Studien belegen übereinstimmend, dass die öffentlich-rechtlichen Fernsehnachrichten die Qualität der Privaten in Sachen Umfang und gesellschaftspolitischer Relevanz deutlich übersteigen (für einen Überblick Daschmann 2009, 260-264; vgl. Betz 2015). Der Journalismus der privaten Vollprogramme legt mehr Wert auf Unterhaltung und Emotionen, tendenziell lässt sich eine »Marginalisierung der politischen Nachrichtengebung « konstatieren (Weiß 2007, 61). Gregor Daschmann $(2009,264)$ ist daher der Auffassung, dass ohne öffentlich-rechtliche Nachrichtenprogramme »derzeit die von der Gesellschaft eingeforderte Qualität des Fernsehnachrichtenangebotes in Deutschland nicht mehr gewährleistet« ist. Allerdings sollten Vergleiche nicht darüber hinwegtäuschen, dass sich der Journalismus des öffentlich-rechtlichen Rundfunks seit der Einführung des »dualen Systems « dem der Privaten annähert. Wolfgang Donsbachs und Katrin Büttners $(2005,34)$ Untersuchung der öffentlich-rechtlichen und privaten Fernsehnachrichten von ARD, ZDF, Sat1 und RTL aus den Jahren 1983, 1990 und 1998 kommt zu dem Ergebnis:

Tendenzen zu einer stärker boulevardesken Darstellung der Politik in den Fernsehnachrichten sind unverkennbar. Sie zeigen sich auf allen Ebenen der Boulevardisierung, also in den Inhalten, dem Stil und der Aufmachung. In einzelnen Dimensionen haben sich sogar dramatische Entwicklungen vollzogen, zum Teil bei allen Anbietern, zum Teil nur bei den Privaten.

Demnach ist das Volumen politischer Themen stark gesunken und die Berichterstattung enthält zunehmend personenbezogene, emotionale, skandalträchtige, sensationelle und spekulative Elemente (ebd., 34). Diese Merkmale sind zwar einerseits bei den privaten stärker ausgeprägt als bei den öffentlich-rechtlichen Sendern, andererseits sind die Befunde der Studie von Donsbach und Büttner aber »eher pessimistisch «, was die »Qualität medialer Vermittlung von politischer Wirklichkeit« sowie die »Erfüllung öffentlicher Aufträge durch die Fernsehsender« angeht (ebd., 34-35):

Politik wird offensichtlich immer seltener Gegenstand von Nachrichten, und sie wird, wenn sie vorkommt, zunehmend in einer Art und Weise präsentiert, die sie möglicherweise dem breiten Publikum schmackhafter und konsumierbarer macht, die aber immer weniger in der Lage ist, ein kohärentes und wirklichkeitsadäquates Bild zu zeichnen. (ebd., 35)

Ein Gutachten für die Linke-Fraktion im Bundestag von Jörg Becker et al. (2007) bilanziert dann auch einen allgemeinen Qualitätsverlust bei 
den Öffentlich-Rechtlichen. Demnach verzeichneten ARD und ZDF schon seit Beginn ihres Sendestarts einen Rückgang bei den Informationssendungen (ebd., 12). Die Forscher stellen außerdem eine Abnahme von Sendungen mit politischen Themen zugunsten eines »Anstiegs an Boulevardisierung « (ebd.) fest. Ähnlich wie Donsbach und Büttner kommen Becker et al. zu dem Schluss, dass diese Entwicklungen eine »erhebliche Relevanz für den Grundversorgungsauftrag des öffentlich-rechtlichen Rundfunks« haben (ebd.).

Dazu kommt eine Politisierung der für Programmkontrolle und Rundfunkaufsicht zuständigen Rundfunk- und Fernsehräte (ebd., 3031). Den Gremien mangelt es oft an professioneller Fachkompetenz, Effektivität und externer Kontrolle; des Weiteren haben die politischen Parteien überproportionalen Einfluss, sodass viele Rundfunk- und Fernsehräte ihrer Kontrollfunktion kaum noch nachkommen können (ebd.; Deutscher Bundestag 2009, 9-10). Letzteres bestätigt auch eine Studie zur Zusammensetzung der Rundfunkräte des Rundfunks BerlinBrandenburg (RBB), des Bayerischen Rundfunks (BR) und von Radio Bremen (RB) durch die Zeitschrift Liberal, die von der FDP-nahen Friedrich-Naumann-Stiftung herausgegeben wird. Die Mehrheit aller Mitglieder (zwischen 58 und 67 Prozent) der untersuchten Rundfunkräte waren entweder Staatsvertreter*innen und Vertreter*innen von Kommunen sowie staatlichen Einrichtungen oder Entsandte von Institutionen, die staatliche Finanzhilfe empfangen (Eichler 2013, 84-87). »Der Rundfunk in Deutschland ist vom Ziel der Staatsferne weiter entfernt denn je«, heißt es in der Studie, »[d]enn die unabhängigen Vertreter der sogenannten sgesellschaftlich relevanten Gruppen werden oft von Institutionen entsandt, die von staatlicher Finanzierung abhängig sind « (ebd., 84).

Zusammenfassend kann festgehalten werden: Der Substanzverlust des Zeitungssektors sowie die Boulevardisierung und Instrumentalisierung des Rundfunks haben auf allgemeiner Ebene zu einer Synchronisierung des Journalismus geführt. Wie Marcus Klöckner $(2019,158)$ beobachtet, »erblinden die Wächter der Demokratie zunehmend und werden immer unfähiger, zentrale politische Weichenstellungen mit dem notwendigen Druck und auch mit der notwendigen Schärfe zu kritisieren«.

Der zunehmende Qualitätsverlust der Nachrichten ist auch ein Resultat der Triebkräfte und Einflusselemente der kapitalistischen Produktionsweise $(\mathrm{kPw})$, denen Journalist*innen und Nachrichtenmedien genauso wie Werbetreibende, Manager*innen und Medieneigen- 
tümer*innen als Teil der Industrie unterworfen sind (Knoche 2013, 91). Nach Manfred Knoche fallen darunter »die strukturell bedingten Mechanismen « der kapitalistischen Medienproduktion: Profitmaximierung auf Basis von Privateigentum an Produktionsmitteln, Mehrwertproduktion, Konkurrenz und Kapitalakkumulation (ebd.). Sie begrenzen den Handlungsspielraum von Medienorganisationen und ziehen auch die öffentlich-rechtlichen Sender in ihr Einflussfeld (ebd.). Knoche (ebd., 92) hat diesen Prozess wie folgt beschrieben:

Aus der Sicht der Kapitaleigner kommt es deshalb auch elementar darauf an, durch geeignete Modifikationen der kPw gegen ihre Interessen gerichtete Transformationen zu verhindern. Sie initiieren stattdessen grundsätzlich nur Restrukturierungen und Transformationen, mittels derer die Kapitalverwertung optimiert sowie das Kapitalverhältnis und die kapitalistische Gesellschaftsformation gesichert werden. Vorangetrieben wird damit der Prozess der formellen und reellen Subsumtion der Gesellschaft unter das Kapital. (Hervorhebung im Original)

Das heißt: Kapitalverschiebungen durch die Werbetreibenden weg von Nachrichtenmedien hin zu Internetmonopolisten machen aus unternehmerischer Perspektive Sinn, da Google und Facebook einen direkteren Zugang zu klar definierten Zielgruppen bieten. Eigentümer*innen und Leiter*innen von Zeitungsunternehmen sind dazu gezwungen, Werbeverluste auch durch Redaktionsverschlankung und Rationalisierung der journalistischen Arbeitsweise zu kompensieren. Die Boulevardisierung und Entpolitisierung der Fernsehnachrichten resultieren ebenfalls aus dem Wettbewerb zwischen öffentlich-rechtlichen und privaten Rundfunkanstalten, bei dem es für die Manager*innen und Kapitaleigner*innen um Reichweiten geht. Obwohl die Politisierung der Rundfunkräte kein direktes Resultat der $\mathrm{kPw}$ darstellt, führt sie auch zu einer weiteren Entfesselung der kapitalistischen Handlungslogik innerhalb des öffentlich-rechtlichen Rundfunks. Denn die bürgerlichen Parteien, die dort einen überproportionalen Einfluss ausüben, befinden sich im Griff der $\mathrm{kPw}$. Das zeigt ein Blick auf die derzeitige Medien- und Wirtschaftspolitik, die sich vor allem durch Privatisierung, Deregulierung und Konzentrationsförderung kennzeichnet (vgl. Knoche 2013, 91).

Was bei diesen Prozessen auf der Strecke bleibt, ist das von der Gesellschaft geforderte öffentliche Gut: Für den Journalismus sind nur noch unzureichend Ressourcen und Anreize vorhanden. Der britische Journalist Nick Davies hat in einer Studie gezeigt, wie die Realität im Geburtsland des Kapitalismus heute aussieht: Journalist*innen müssen im 
Durchschnitt 48 Berichte pro Woche schreiben, das sind 9,6 an einem Tag $(2009,59)$. Die Arbeit wird hauptsächlich vom Schreibtisch aus erledigt: Von 45,5 Arbeitsstunden pro Woche entfallen lediglich drei auf Recherchen »außer Haus«, während denen sich Journalist*innen im Durchschnitt mit vier Interviewpartnern*innen treffen (ebd.). Als Ergebnis dieser Arbeitsverhältnisse bestehen 60 Prozent aller Nachrichtenbeiträge der Qualitätsmedien hauptsächlich aus Agentur- oder PR-Material (ebd., 52). Davies (2008) macht eine fortschreitende Kommerzialisierung für diesen Zustand verantwortlich: Kürzungen in den Redaktionen durch die Finanzplaner*innen der Konzerne hätten Journalist*innen zu passiven Verarbeiter*innen von Informationen aus zweiter Hand gemacht. Daher fänden sich heute selbst die besten und anständigsten ihrer Zunft in einer Art Käfig gefangen (ebd.). Medienökonomisch gesprochen ist diese Aushöhlung des Journalismus, die auch in Deutschland weit vorangeschritten ist, als ein externer Effekt des Marktes anzusehen.

\section{Institutionen der kapitalistischen Medienindustrie}

In diesem Abschnitt werden auf Basis der kritischen politischen Ökonomie der Medien (Curran 2002; Ferschli, Grabner und Theine 2019; Herman 1995; 1999; Knoche 2013; McChesney 2008; Zollmann 2009; 2017) und der Medienökonomie (Heinrich 2002; Kiefer 2001) die relevanten Institutionen aufgezeigt und deren Probleme für die Nachrichtenproduktion veranschaulicht. Diese Vorgehensweise verdeutlicht den Zusammenhang zwischen der Krise des Journalismus und den kapitalistischen Strukturen der Nachrichtenmedien.

\section{1 Privateigentum/Konzernstruktur/Profit}

Die zehn größten deutschen Medienunternehmen sind als privatwirtschaftlich agierende Medienkonzerne (Kapitalgesellschaften) organisiert, die von einzelnen Familien oder einem Familiengeflecht kontrolliert werden. Die beiden einzigen Ausnahmen bilden die ProSiebenSat.1-Gruppe, deren Anteile verschiedenen internationalen Finanzinvestoren gehören, und der gebührenfinanzierte, öffentlichrechtliche Rundfunk (Ferschli et al. 2019, 23-24). Die meisten Zeitungsverlage außerhalb der Top 10 der Medienunternehmen sind ebenfalls als private Konzerne organsiert. 
Auf einer allgemeinen Ebene sind Konzerne im Kapitalismus als Institution juristisch dazu verpflichtet, ihrem eigenen organisatorischen und nicht etwa dem gesellschaftlichen Gemeinwohl zu dienen (Bakan 2005, 1-2). Das bedeutet: Medienkonzerne müssen in erster Linie, und im Sinne ihrer Anteilseigner*innen, Profite maximieren. Journalistische und andere von der Gesellschaft an die Medien gestellte Imperative sind nicht Teil der Handlungslogik und bestenfalls als sekundär zu betrachten. Wenn Medienunternehmen zu Konglomeraten fusionieren, Redaktionen zusammenschließen, auf Mantelproduktion umsteigen, Arbeitsabläufe rationalisieren oder Personal abbauen, ist das aus unternehmerischer Perspektive sinnvoll, weil dabei gemäß der institutionellen Handlungslogik Profite generiert werden (für eine allgemeine Diskussion dieser Sachverhalte mit Blick auf die Handlungslogik von Konzernen vgl. Bakan 2005; Zollmann 2017).

Konzerneigentümer*innen und Manager*innen sind außerdem Teil der Wirtschaftsgemeinde, sie verkehren in gemeinsamen Gremien und sozialen Kreisen. Durch Anteilseignung und Kreditvergabe nehmen andere Unternehmen und Banken Einfluss auf Medienkonzerne. Interessenkonflikte werden durch solche Verflechtungen institutionalisiert (Zollmann 2009, 112).

\subsection{Markt und Wettbewerb}

Konzernmedien verkaufen ihre Medienprodukte auf zwei Märkten: an die Werbeindustrie und an Rezipient*innen. Diese nehmen auf verschiedenen Ebenen Einfluss auf die Nachrichtenproduktion:

\section{Kosteneffizienz}

Einerseits stehen Nachrichtenmedien konstant unter Druck, möglichst kostengünstig zu produzieren, und sind ökonomischen Schwankungen wie auch Krisen ausgesetzt. Andererseits kosten journalistische Recherchen zu politischen oder wirtschaftlichen Themen teures Geld: Personal-, Zeit- und Rechercheaufwand sowie das Risiko, verklagt zu werden, müssen einkalkuliert werden. Marktwirtschaftliche Zwänge veranlassen die Eigentümer*innen und Manager*innen von Nachrichtenorganisationen dazu, eine publizistische Strategie zu wählen, die es ermöglicht, die Kosten zu minimieren. Diese Tendenz wird durch das in Medienkonzernen institutionali- 
sierte Profitimperativ verstärkt. PR- und Agenturmaterial erhält daher Vorrang vor investigativen Recherchen zu sensiblen Themen (Zollmann 2017, 40).

Die im letzten Abschnitt angesprochenen Einbrüche im Werbemarkt haben den ökonomischen Wettbewerb um Werbekund*innen und Leser*innen verschärft. Viele Zeitungsverlage mussten ihre Verkaufspreise anheben, um Verluste zu kompensieren, was wiederum die Gefahr, Käufer*innen zu verlieren, nach sich zog (Röper 2020, 333). Gleichzeitig haben sie seit Jahren mit Auflagenrückgang und sinkenden Gesamteinnahmen zu kämpfen (ebd., 331-333). Das alles begünstigt weitere Einsparungen, die zu Lasten der journalistischen Qualität gehen. Die Gravitation des Marktes und der Wettbewerb mit den Privaten beeinflussen auch die öffentlich-rechtlichen Sender. Die in Abschnitt 2 untersuchte Boulevardisierung und Entpolitisierung der Fernsehnachrichten sind ebenfalls auf Marktzwänge zurückzuführen, denn boulevardeske Nachrichten sind kostengünstig und versprechen hohe Reichweiten.

\section{Konzentration}

Eine weitere durch Marktzwang begünstigte Strategie von Medienkonzernen ist, über Zusammenschlüsse und Fusionen Größenvorteile zu erlangen. Das erlaubt es, Inhalte mehrfach zu verwerten und anderen Anbietern den Zutritt zum Werbe- und Publikumsmarkt zu erschweren.

Die Struktur aller deutschen Medienmärkte weist ein hohes Maß an Konzentration auf und kann als ein Oligopol bezeichnet werden. Auf lokaler und regionaler Ebene gibt es sogar Monopole im Zeitungssektor. Das bedeutet: Wenige große Unternehmen beherrschen die Medienmärkte im Presse-, Rundfunk- und Onlinesektor auf Seite der Anbieter und Rezipient*innen (Ferschli et al. 2019, 12). Diese so gut wie alle kapitalistischen Mediensysteme kennzeichnende Konzentration hat schwerwiegende Folgen für die publizistische Vielfalt (Fenton et al. 2020, 85; Leidinger 2003). Liberale Pressetheorien gehen davon aus, dass eine freie Meinungsbildung nur dann möglich ist, wenn Rezipient*innen vielfältige Meinungen und Informationen zur Verfügung stehen (Alexander 1981). Dieses Prinzip wird durch Medienkonzentration ausgehebelt, denn die als Oligopolisten agierenden Konzerne bieten lediglich eine ideologisch begrenzte Menge von »Meinungsoptionen« zur Auswahl (Herman 1999, 295). Dadurch fallen eine Vielzahl von Positionen unter den Tisch und der freie Austausch von Informationen wird behindert. 
Auch agieren Oligopolisten durch ihre Werbereichweiten und crossmedial aufgebauten Portfolios als Marktschranken. Ein wichtiges Beispiel ist das Internet, in dem professionelle journalistische Angebote überwiegend von traditionellen Medienunternehmen erstellt werden (siehe Lobings und Neuberger 2018, 65, 160). Dort gibt es also so gut wie keine relevanten und reichweitenstarken journalistischen Anbieter, die nicht Teil der krisenhaften, oligopolistischen Struktur der Nachrichtenmedien sind (siehe Abschnitt 2).

\section{Ausgrenzung von Zielgruppen}

Marktabhängigkeit hat auch direkte ideologische Effekte für die Medieninhalte. Auf dem Zuschauer*innenmarkt werden eher wohlhabendere Zielgruppen, insbesondere Akademiker*innen und professionelle Eliten, anvisiert. Diese Marktsegmente können sich den Verkaufspreis leisten und werden von der Werbeindustrie wegen ihrer Kaufkraft bevorzugt. Die Lebenswelten von Arbeiter*innen, Minderheiten oder Geflüchteten finden daher kaum Berücksichtigung.

Die Werbeindustrie fungiert außerdem als indirekte Lizensierungsaufsicht der privaten Medien (Curran 2002, 96), da Werbekund*innen nur in solchen Nachrichtenmedien Werbung schalten, deren Medieninhalte ihren ideologischen Präferenzen entsprechen (Herman 1995, 169). Historisch hatte das zur Folge, dass Medienunternehmen, die inhaltlich die Interessen der Arbeiterklasse vertraten und ein großes Publikum ansprachen, vom Markt verdrängt wurden, weil sie nicht genug Werbeeinnahmen akquirieren konnten (Curran 2002, 81-103).

\section{Externe Effekte}

Nachrichten sind sogenannte öffentliche oder meritorische Güter, deren Allokation über Märkte zu externen Effekten führt. Diese umfassen, was die Medien angeht, Boulevardisierung, Entpolitisierung oder Fehlinformierung.

Idealtypisch sollten Angebot und Nachfrage die Produkte an die Präferenzen der Rezipient*innen anpassen und eine optimale Versorgung bieten. Weil Nachrichten aber ständig neu produziert werden, fehlt den Rezipient*innen das relevante Wissen, um die Qualität und Richtigkeit der Nachrichten überprüfen zu können (Kiefer 2001, 136-139). Anders ausgedrückt: Wenn Nachrichten eine bestimmte ideologische Sichtweise hervorheben, über wichtige Ereignisse überhaupt nicht berichten oder zu wenig Informationen liefern, ist das als Rezipient*in nur schwer nachvollziehbar. Diese Mängel führen bei öffentlichen Gütern 
dazu, dass keine ausreichende Bandbreite an relevanten Informationen angeboten wird. Daher kommt es zu externen Effekten, im Zuge derer die Rezipient*innen nur ungenügend oder sogar fehlinformiert werden. Deshalb ist es in der Medienökonomie unumstritten, dass ein Marktsystem nicht in ausreichendem Maße die Produktion und Konsumption meritorischer Güter wie Nachrichten gewährleisten kann (Heinrich 2002, 46; Kiefer 2001, 136-148). Jürgen Heinrich (2002, 46) argumentiert beispielsweise, dass die Produktion von Öffentlichkeit, Meinungsvielfalt, Wahrheit und gesellschaftlicher Normen »nicht dem Markt überlassen« werden sollte. Denn diese Bereiche »sind nur jenseits der Kategorien von Gebrauchswert und Tauschwert zu bewerten «, liegen »mithin normativ jenseits der Wirkungsweise des Wettbewerbs « und sind seiner theoretisch fundierten ökonomischen Analyse im Grunde nicht zugänglich« (ebd.).

Die Gesellschaft muss daher eingreifen, um eine Grundversorgung mit Nachrichten oder politischen Themen zu gewährleisten.

\subsection{Produktionsverhältnisse und professioneller Journalismus}

Die hier dargelegten Institutionen und Tendenzen definieren die Produktionsverhältnisse in der kapitalistischen Medienindustrie. Die kritische politische Ökonomie sieht des Weiteren diese »Produktionsverhältnisse als Herrschaftsverhältnisse und darauf basierendes Handeln im Interesse der Kapitaleigner als bestimmende Triebkraft für die Entwicklung der Produktivkräfte (Produktionsmittel und Arbeit)« (Knoche 2013, 94).

Das heißt: Professionelle Journalist*innen müssen sich diesen institutionellen Zwängen anpassen. Die Unabhängigkeit und Integrität des Journalismus sollen zwar durch eine Berufsethik und einen professionellen Kodex gewährleistet werden, aber - wie zahlreiche Studien belegen - ist es in der Realität kaum möglich, der Marktlogik zu entkommen (Zollmann 2009; 2017 für einen Überblick). Daher muss eine Reform an den Wurzeln ansetzen und die Strukturen der Medien verändern. 


\section{Visionen und Strategien der Medienreform}

Im Folgenden wird erläutert, von welchen normativen Werten eine Medienreformbewegung geleitet sein sollte und mit was für kurz-, mittel-, und langfristigen Maßnahmen sich strukturelle Reformen umsetzen lassen. ${ }^{2}$ Eine Transformation der Medien ist notwendig, damit von der Gesellschaft gewünschte Funktionen des Journalismus, wie Meinungsvielfalt, Wahrheitssuche oder objektive Informationsvermittlung, gewährleistet werden können.

\subsection{Normativer Werterahmen: Medienreform bedeutet Mediengerechtigkeit}

Der Begriff »Reform《 sollte zum weiteren Verständnis kurz beleuchtet werden. Denn die hier vorgestellten Werte und Maßnahmen haben nichts mit den in den letzten Jahrzehnten in vielen Demokratien durchgeführten neoliberalen »Reformen«, die einen technokratischen Charakter hatten mit dem Ziel, kosmetische Veränderung oder Privatisierungen von Medien durchzuführen, zu tun (Fenton et al. 2020, 28). Fenton et al. schlagen daher vor, nicht von Medienreform, sondern von Mediengerechtigkeit zu sprechen. Darunter fallen »underlying struggles of class, gender and race that are at stake when we talk about the need for more balanced, pluralistic, representative, accurate and accountable sources of news and information, or a more accessible, diverse and participatory system of cultural production « (ebd.).

Diese Sichtweise impliziert, dass auf marktwirtschaftlichen Annahmen basierende Ansätze der Transformation der Medien fehlgeschlagen sind und nicht einmal in der Lage waren, liberale Basisziele wie die Gewährleistung von Wettbewerb und Privatsphäre oder Vielfalt und Freiheit zu erreichen (ebd.). Mediengerechtigkeit versucht demnach,

2 Hier wird im Wesentlichen auf eine Reform der Nachrichtenmedien und des Journalismus eingegangen. Reformen von nichtjournalistischen Medien insbesondere im Internet (Soziale Medien, Suchmaschinen, Buchhandel und weitere) werden hier aus forschungsökonomischen Gründen nicht direkt berücksichtigt. Sicherlich sollte eine umfassende Medienreform auch bei den Internetkonzernen ansetzen, die quasi als Monopolisten agieren und bei der Nachrichtenverbreitung eine Rolle spielen. Einige der hier vorgeschlagenen Reformmaßnahmen (zum Beispiel die Konzentrationskontrolle und Besteuerung von Werbung) könnten dort ebenfalls angewandt werden. 
nicht nur Abhilfe gegen die in Abschnitt 2 beschriebene Aushöhlung des Journalismus zu schaffen, sondern auch Machtungleichheiten zu beseitigen, die auf Kosten von Arbeitern*innen, Frauen und Minderheiten gehen. Die sich aus Kapitalismus und Marktlogik ergebene Akkumulation von Macht in der Hand weniger Konzerne und Eigentümer*innen ist aus dieser Perspektive ein Kernproblem der Medien (ebd., 28-29). Daher müssen, wie Fenton et al. abschließend argumentieren, Strategien der Medienreform von Prinzipien wie Egalitarismus, Gerechtigkeit und Demokratie sowie finanzieller und ökologischer Verantwortung geleitet werden und als Teil von visionären und emanzipatorischen Bewegungen der Zivilgesellschaft, die für soziale, politische, wirtschaftliche und ökologische Transformation einstehen, ablaufen (ebd., 84).

\subsection{Verzahnung von Visionen und Strategien des Medienwandels}

Zusätzlich zu visionären Überlegungen bezüglich der institutionellen Basis eines reformierten Mediensystems brauchen Reformbestrebungen eine Strategie, wie Veränderungen realistisch erreicht werden können. Außerdem gilt es zu evaluieren, welche bewährten Aspekte des Mediensystems beizubehalten und welche zu verändern oder auszutauschen sind (McChesney 2008, 491). Medienreformbestrebungen enthalten daher ein Bündel von kurz-, mittel- und langfristig durchsetzbaren transformativen, visionären und strategischen Elementen. Darauf aufbauend sollten die Konturen neuer Institutionen entwickelt und in die Langzeitstrategie für Medienwandel integriert werden. Auch muss die Medienreformbewegung kontinuierlich ihre Visionen und Strategien evaluieren und gegebenenfalls revidieren und erneuern.

Die hier vorgeschlagene Medienreform hat zum Ziel, positive Aspekte des bestehenden Fundaments der Medien zu stärken und nicht einzureißen. Sie baut auf existierende normative und gesetzliche Rahmenbedingungen der Presse und des Rundfunks auf. Dazu gehören beispielsweise die ethischen Standards des Journalismus wie der vom Presserat erstellte Pressekodex, die beibehalten und erweitert werden sollten. Gleiches gilt für den Auftrag des öffentlich-rechtlichen Rundfunks, der sich aus dem Grundgesetz und Rundfunkstaatsvertrag ergibt und wichtige Momente wie »Grundversorgung «, »Ausgewogenheit« oder »Überparteilichkeit« enthält. 
Die Medienreform vollzieht sich, ähnlich wie andere gesellschaftliche Transformationen, als ein langfristiger Prozess, der von sozialen Bewegungen, Zivilgesellschaft, Wissenschaft und Regulierungsbehörden mit dem Ziel vorangetrieben werden muss, die Politik zum Handeln zu bewegen (McChesney et al. 2005). Wie die Arbeit von Mandy Tröger $(2019,117,133)$ zeigt, folgt die ordnungspolitische Regulierung (und die Kommunikationswissenschaft) in Deutschland allerdings einem ideologischen Mantra, das Pressefreiheit mit privatwirtschaftlicher Organisation gleichsetzt. Demnach ermöglicht Medienpolitik Transformationen, wenn diese mit Privatisierung und Kommerzialisierung einhergehen. Auf der anderen Seite stoßen Reformvorschläge, die mehr öffentliche Kontrolle der Medien fordern, auf Widerstand. Denn Ordnungspolitik wird von der neoliberalen Doktrin geleitet und Medienkonglomerate sind in der Lage, die Politik in ihrem Interesse zu beeinflussen und mitzugestalten.

Daher können die hier vorgeschlagenen Reformmaßnahmen, die öffentlich finanzierte Medien substanziell stärken möchten, nur durch Unterstützung einer starken Medienreformbewegung umgesetzt werden. Um substantielle Veränderungen zu erreichen, muss ein öffentliches Bewusstsein dafür geschaffen werden, warum eine Reform nötig ist (Stichwort: Krise des Journalismus) und welche Strategien dabei helfen, diese politisch umzusetzen (McChesney 2005, 12-13). Zivilgesellschaftliche Akteur*innen, die als Vermittler*innen der Medienreform auftreten, sollten außerdem in der Lage sein, Debatten zu framen und medienpolitische Entscheidungen anzustoßen. Das muss insbesondere auf Länderebene geschehen, weil dort wichtige Aspekte der Mediengesetzgebung stattfinden, sollte aber auch auf Bundesebene ablaufen, damit Kernreformen länderübergreifend umgesetzt werden können.

Grundsätzlich sollten die Reformbestrebungen auch im Medienrecht verankerte Anweisungen von Regulierungsstellen zur Hilfe nehmen. Denn wichtige Normen, zum Beispiel zur Vielfaltssicherung, sind bereits durch das Bundesverfassungsgericht, den Europäischen Gerichtshof und in Rundfunkstaatsverträgen kodifiziert (KEK 2018, 46-48). Die Kommission zur Ermittlung der Konzentration im Medienbereich (KEK) hat Gutachten zur Konzentrationskontrolle inklusive zeitgemäßer Reformanweisungen verfasst, welche die Gesetzgeber*innen aber bestenfalls schwerfällig umsetzen (ebd., 488-498). Eine entsprechende Bewegung kann sich diese Instrumente zu eigen machen: Wenn es gelingt, genug öffentlichen Druck auf die Politik aufzubauen, kön- 
nen die von diesen Institutionen bereits vorgebrachten Reformansätze realisiert werden. ${ }^{3}$

\subsection{Kurz- und mittelfristige Medienreformmaßnahmen}

Im Folgenden werden Reformmaßnahmen aus ausgewählten Studien (Becker et al. 2007; Beiler und Krüger 2018; Fenton et al. 2020; Herman 1999; KEK 2018) herausgearbeitet, die kurz- und mittelfristig eingeleitet und mit Blick auf die deutsche Medienlandschaft realisiert werden können. Medienreformen werden demnach unter den folgenden Aspekten zusammengefasst: Konzentrationskontrolle, unabhängige Aufsicht und Überwachung journalistischer Standards, Demokratisierung der Rundfunkräte sowie Aufbau und Finanzierung unabhängiger Nachrichtenmedien.

\section{Konzentrationskontrolle}

Die Schaffung einer medienübergreifenden Konzentrationskontrolle (Gesamtmarktmodell) unter Aufsicht der KEK ist dringend erforderlich. Sie bemerkte diesbezüglich in ihrem letzten Gutachten, dass die derzeitige Umsetzung lediglich am Zuschauer*innenmarkt des privaten Fernsehens ansetze, es aber einer Betrachtung des Gesamtmedienmarktes bedürfe, um allen »meinungsbildenden Faktoren Genüge zu tun« und die »adäquate Erfassung und Bewertung von Verflechtungen« zu ermöglichen (KEK 2018, 492-493). Die in Abschnitt 3.2 angesprochenen Konzentrationstendenzen, die sich auf alle Mediensektoren übergreifend erstrecken, müssen in diesem Sinne entschärft werden. Dafür bedarf es auch der Erstellung neuer Richtlinien, ab welchem Grad Verflechtungen der Meinungsbildung abträglich sind. Denn die

3 Natürlich sollten Akteur*innen der Medienreformbewegung die rechtlich und regulativ kodifizierten Medienreformvorschläge auch evaluieren und gegebenenfalls Debatten anstoßen, wie diese im Sinne von Mediengerechtigkeit zu verbessern wären. Ein Beispiel einer Reformbewegung ist die britische »Media Reform Coalition« (MRC), ein Zusammenschluss aus Wissenschaftler*innen, politischen Aktivist*innen und Gruppen der Zivilgesellschaft, der das gemeinsame Ziel einer progressiven Medienreform verfolgt (Fenton et al. 2020, 7). Die MRC erstellt Analysen zur Medienkonzentration, engagiert sich bei öffentlichen Veranstaltungen, bietet Lageberichte für parlamentarische Untersuchungen und entwickelt Vorschläge, wie die Medien im öffentlichen Interesse verbessert werden können. 
Instrumente und Richtwerte der derzeitigen Konzentrationskontrolle können im Licht des realen Ausmaßes kaum als ausreichend bezeichnet werden. ${ }^{4}$

Konzentrationskontrolle ist des Weiteren unzureichend, wenn sie nur "passiv«, also bei zu hohen Konzentrationswerten, greift. Das heißt: Vielfalt sollte nicht nur im Hinblick auf die Zahl der Anbieter gesichert werden; Konzentrationskontrolle muss auch »aktiv« eine reichhaltige Medienlandschaft fördern. Das kann durch die Etablierung neuer öffentlich-rechtlicher, unabhängiger (gemeinnütziger) und privater Anbieter auf lokaler, regionaler und nationaler Ebene geschehen (Fenton et al. 2020, 86). Auch hierzu sagte die KEK (2018, 493): »Effektive Vielfaltsicherung ist gleichermaßen negativ abwehrend und positiv gestaltend anzulegen und am Verfassungsgebot des Art. 5 Abs.1 GG auszurichten.« Allerdings beschränke sich die derzeitig gesetzlich vorgeschriebene Konzentrationskontrolle lediglich auf defensive, negative Vielfaltssicherung (ebd.). Eine Änderung des Staatsvertrags dahingehend, dass die KEK auch strukturgestaltend tätig werden darf, gilt es bei den Gesetzgeber*innen durchzusetzen. So könnte sie Befugnisse erhalten, Medieneinheiten ab einer gewissen Konzentrationsschwelle zu spalten und in kleinere, voneinander unabhängige Organisationen aufzuteilen. Im Dienste einer positiven Gestaltungsfunktion muss die KEK außerdem das Mandat und die Mittel erhalten, den Aufbau neuer Medienorganisationen voranzutreiben. Dann könnte sie zum Beispiel mittels Ausschreibeverfahren für Lizenzen und der Vergabe kompetitiver Fördermittel die Medienlandschaft aktiv mitgestalten.

4 Daten für die Auflagen von Tageszeitungen werden beispielsweise lediglich bundesweit veranschlagt (Ferschli, Grabner und Theine 2019, 13). Die nationalen Konzentrationsmessungen sind also blind gegenüber einer erheblichen Zahl von lokalen oder regionalen Zeitungsmonopolen (ebd.). Laut dem Rundfunkstaatsvertrag könnte eine vorherrschende Meinungsmacht im Fernsehsektor bestehen, wenn auf die Programme einer Sendergruppe ein Zuschauer*innenanteil von 30 Prozent oder mehr entfällt (KEK 2018, 48). Demnach werden die Verhältnisse deutscher Fernsehsender als angemessen eingestuft. Dabei dominierte 2018 ein Oligopol bestehend aus den öffentlich-rechtlichen Sendern mit insgesamt 48,2 Prozent (ARD: 11,5; ZDF: 13,9; andere Öffentlich-Rechtliche: 22,8), der RTL Group mit 22,0 Prozent und der ProSiebenSat.1 Media AG mit 17,9 Prozent den Markt der Fernsehzuschauer*innen in Deutschland (Ferschli, Grabner und Theine 2019, 15). Die Richtwerte der Konzentrationskontrolle ermöglichen damit ein mächtiges Rundfunkoligopol. 
Unabhängige Aufsicht und Überwachung journalistischer Standards Von der Industrie und Regierung unabhängige Rundfunk- und Presseräte sollten dafür zuständig sein, die Kodifizierung und Einhaltung von Rundfunk- und Pressestatuten zu überprüfen, ethische Standards aufrechtzuerhalten, öffentliche Beschwerden abzuwickeln und gegebenenfalls Sanktionen bei Fällen einer Fehlberichterstattung auszusprechen, damit die Integrität des Journalismus gewährleistet bleibt (Fenton et al. 2020, 89). Hier könnte die Medienreformbewegung bei den bestehenden Rundfunk- und Presseräten sowie Landesmedienanstalten (zuständig für die privaten Sender) ansetzen, die im derzeitigen System für Beschwerden zuständig sind. Wie schon in Abschnitt 2 besprochen, werden die Rundfunkräte von den politischen Parteien dominiert. Die Organe des Presserats setzen sich im Wesentlichen aus Vertreter*innen der Organisationen Bundesverband Digitalpublisher und Zeitungsverleger (BDZV), Deutscher Journalisten-Verband (DJV), Deutsche Journalistinnen- und Journalisten-Union (dju) in ver.di und Verband Deutscher Zeitschriftenverleger (VDZ) zusammen. ${ }^{5}$ Es fehlt daher die nötige Unabhängigkeit von der Industrie. Rundfunk- und Presseräte sowie die Räte der Landesmedienanstalten müssen daher im Sinne einer repräsentativen Bürger*innenbeteiligung reformiert werden.

Auch gilt es, die Befugnisse und Sanktionsmittel dieser Organe auszuweiten. Denkbar wäre beispielsweise eine aktivere Rolle der Behörden: So könnten Medieninhalte in Bezug auf die Einhaltung journalistischer Standards regelmäßig überprüft und Ergebnisse dieser Untersuchungen in kontinuierlichen Abständen veröffentlicht werden. Dies ließe sich auch über eine Bürger*innenbeteiligung, beispielsweise durch die Errichtung einer Stiftung Mediawatch, die Gutachten zur Berichterstattung für die Rundfunk- und Presseräte erstellt, umsetzen (Becker et al. 2007, 52). Was Sanktionen angeht, sollten Medienorganisationen bei aufgedeckten Verletzungen journalistischer Standards dazu verpflichtet werden, einen größeren und prominenteren Raum ihrer Berichterstattung für Richtigstellungen und Gegendarstellungen zur Verfügung zu stellen. Auch müssen die Rundfunk- und Presseräte Fehlverhalten öffentlich thematisieren und missbilligen. Um die Pressefreiheit zu wahren, sollten allerdings keine Sanktionsbefugnisse erteilt werden, die direkt in die Redaktionen eingreifen.

5 https://www.presserat.de/aufgaben-organisation.html. Zugegriffen: 10. November 2020. 
Ein wichtiger Teil der Medienreform bestünde außerdem in einer Erweiterung der ethischen Standards des Journalismus: So könnten redaktionelle Statuten ein Element des Konstruktiven Journalismus enthalten (Beiler und Krüger 2018; Krüger in diesem Band). Dieser fokussiert auf die Lösung von Problemen und vermittelt Strategien, wie Menschen positive gesellschaftliche Veränderungen erreichen können (Beiler und Krüger 2018, 185). Das erscheint auch mit Blick auf den menschengemachten Klimawandel von Bedeutung: Hier könnte Konstruktiver Journalismus eine wesentliche Rolle spielen, indem er »Bürgerinnen und Bürger über Problemlösungsversuche informiert und damit gleichzeitig ermächtigt, gegebenenfalls selbst zielgerichtet tätig zu werden« (ebd., 180). Um solche Reformen politisch umzusetzen, schlagen Beiler und Krüger (ebd., 185) vor, durch neue Vorgaben im Rundfunkstaatsvertrag »den Programmauftrag für die öffentlich-rechtlichen Sender um eine konstruktive Komponente« zu ergänzen. Auf ähnliche Weise könnte man den vom Presserat herausgegebenen Pressekodex, der von den meisten Verlagen anerkannt wird, um eine konstruktive Richtlinie erweitern.

Demokratisierung des öffentlich-rechtlichen Rundfunks

Eine strukturelle Reform der Rundfunkräte kann eine demokratischere Kontrolle- und Programmplanung der öffentlich-rechtlichen Sender ermöglichen: Rundfunkräte und -vorstände aller öffentlich-rechtlicher Medien sollten daher von der Gesellschaft gewählt werden und Vertreter*innen aus der Belegschaft enthalten; auch müssen Quotenregelungen absichern, dass alle relevanten Gruppen inklusive der Arbeiter*innen und neuen sozialen Bewegungen in den Rundfunkräten repräsentiert sind. Damit die Vielfalt der Mitarbeiter*innen des Rundfunks gewährleistet bleibt, sollten Daten zur sozialen Zusammensetzung der Arbeitskräfte regelmäßig im Sinne eines Gleichstellungsmonitorings (das auch sozioökonomische Faktoren miteinbezieht) veröffentlicht und überprüft werden. Schließlich sind Grundsatzentscheidungen zur Programmplanung und zur Einführung neuer Dienste unter Berücksichtigung regelmäßiger, repräsentativer Befragungen der Rezipient*innen zu treffen (Becker et al. 2007, 52; Fenton et al. 2007, 88-89).

Die innere Pressefreiheit sollte durch interne Statuten gewährleistet werden, die Journalist*innen "gegen hierarchischen Druck von oben und kurzfristiges Quotendenken absichern« (Becker et al. 2007, 52). Ein möglichst hoher Grad an gewerkschaftlicher Organisation ist dabei 
nötig, um ihre Verhandlungsmacht gegenüber dem Rundfunkmanagement zu stärken. Des Weiteren wird ein Mechanismus benötigt, der es Journalist*innen erlaubt, auf unethisches Verhalten innerhalb der Organisation aufmerksam zu machen, ohne Sanktionen fürchten zu müssen (Fenton et al., 2020, 89). Das Rundfunkmanagement sollte darüber hinaus dezentralisiert und demokratisch aus der Belegschaft für einen befristeten Zeitraum gewählt werden. Über Vollversammlungen ließe sich eine Plattform schaffen, um über wichtige Belange des Rundfunks zu debattieren und abzustimmen. Diese Maßnahmen könnten den Rundfunk aus dem Klammergriff der Politik und Funktionäre befreien und mehr »Aufklärung, Emanzipation, Partizipation und Demokratisierung« ermöglichen (Becker et al. 2007, 51).

Reformmaßnahmen müssen auch dazu führen, dass finanzielle Ressourcen mit Blick auf das öffentliche Interesse und nicht auf Quoten verteilt werden. Das könnte mit einer Stärkung der Finanzkontrolle des öffentlich-rechtlichen Rundfunks durch den Bundesrechnungshof und die Landesrechnungshöfe einhergehen (ebd., 51). Gleichzeitig sollte der öffentlich-rechtliche Rundfunk komplett auf Werbeeinnahmen, Produktplatzierungen und Outsourcing redaktioneller Arbeit verzichten, damit Interessenkonflikte ausgeschlossen bleiben; betriebswirtschaftliche Imperative des Rundfunkmanagements müssen gesellschaftlichen Zielsetzungen weichen (ebd., 51-52). Schließlich wäre eine Veröffentlichung der Honorare von Manager*innen, Führungspersonen und »Stars" der Unterhaltungssendungen sowie Sitzungsprotokolle der Rundfunkräte und Lizensierungsverfahren zur besseren Transparenz geboten (ebd.).

\section{Aufbau und Finanzierung unabhängiger Nachrichtenmedien}

Neben der Stärkung des bestehenden Rundfunk- und Pressesystems sollte die Entwicklung eines gemeinnützigen, unabhängigen Mediensektors mit alternativen Organisations- und Eigentumsstrukturen (zum Beispiel Kooperativen, Stiftungen oder auf Selbstbestimmung basierende Organisationsformen), geleitet von Imperativen wie Gleichheit, Vielfalt, Mitbestimmung, Kollektivismus und Nachhaltigkeit, gefördert werden (Fenton et al. 2020, 87-90). Die KEK oder eine noch zu gründende Behörde für unabhängige Nachrichtenmedien, deren Gremien die Bevölkerung per Wahl bestimmt, könnte die Befugnisse erhalten, diesen Sektor über Lizenzausschreibungen und Fördermittelvergaben mitzugestalten. Gesellschaftliche Gruppen sollten dabei auch in die Lage versetzt werden, mit neuen Organisationsformen und Medienfor- 
maten zu experimentieren. Der Aufbau und Betrieb eines vielfältigen und lebhaften alternativen Mediensektors benötigt allerdings erhebliche Ressourcen. Nach Edward S. Herman $(1999,303)$ besteht ein wichtiger Aspekt der Medienreformstrategie darin, Lösungen zu finden, wie eine stabile und substantielle Finanzierung unabhängiger Nachrichtenmedien ermöglicht werden kann. Die beiden Hauptquellen dafür seien Besteuerung der Einnahmen kommerzieller Medienunternehmen sowie staatliche Zuschüsse und Subventionen.

So wäre eine Finanzierung der reformierten deutschen Medienlandschaft über folgende Modelle möglich: die Landesmedienanstalten könnten Steuern bei den Privaten abschöpfen und damit beispielsweise die durch Werbeeinbrüche geschrumpfte Presse revitalisieren und neue öffentliche oder alternative Medien aufbauen und fördern; alternativ erhielten alle Rezipient*innen einen festgelegten Betrag von den Landesmedienanstalten, der nur für von gemeinnützigen Anbietern produzierte Nachrichten ausgegeben werden darf (Coupon-System). Finanzielle Mittel für diese Subventionssysteme ließen sich über eine Besteuerung von Firmen generieren, deren Marktanteil auf nationalen Medien-, Suchmaschinen- oder Netzwerkplattformmärkten jenseits der 20 Prozent liegt (Fenton et al. 2020, 87-90). Werbekosten, die in Unternehmen fließen, die keine journalistischen Produkte herstellen, könnten, was die steuerliche Abzugsfähigkeit betrifft, anders eingestuft werden als solche, die journalistischen Angeboten zugutekommen. Auch eine Besteuerung von PR-Ausgaben wäre denkbar, denn oftmals bedient sich und profitiert Öffentlichkeitsarbeit vom Journalismus (Kiefer 2011, 17). Darüber hinaus könnten unabhängige Nachrichtenmedien mit eigener journalistischer Redaktion Steuererleichterungen für ihre kultur-gesellschaftliche Leistung erhalten (ebd.). Auch wären die Landesmedienanstalten in der Lage, eine Gebrauchssteuer auf die Nutzung der Übertragungswege Kabel, Satellit, DVBT und IPTV sowie auf Mobil- und andere Telefonverbindungen zu veranschlagen, die private Anbieter nach der Lizensierung zu entrichten haben; denn bei diesen Kommunikationsinfrastrukturen handelt es sich um öffentliche Ressourcen, deren Besteuerung legitim ist (Herman 1999, 303). Schließlich rechtfertigt die gesellschaftliche Bedeutung unabhängiger Nachrichtenmedien mit Blick auf die demokratische Meinungsbildung und Informationsvermittlung die Verwendung allgemeiner Steuern als Mittel der Subvention (ebd.). 


\subsection{Konturen eines reformierten Mediensystems: \\ Langfristige Medienreform bedeutet öffentliche Kontrolle und journalistische Selbstbestimmung}

Die in Abschnitt 3 vorgenommene Analyse der Institutionen der kapitalistischen Medien legt nahe, dass die Nachrichtenproduktion größtenteils von einem System geleistet werden muss, das von wirtschaftlichen und staatlichen Interessen unabhängig zu organisieren ist. Im Umkehrschluss sollten daher Privateigentum, Konzernorganisation, Profit, Markt und Wettbewerb langfristig keine wesentliche Rolle für die Nachrichtenmedien spielen. Robert W. McChesney $(1997,66)$ argumentiert daher, ein Großteil der Nachrichtenmedien müsse unter öffentliche Kontrolle gestellt werden und dürfe nicht von Wirtschaftsunternehmen oder Werbefinanzierung abhängig sein. Um das zu erreichen, können die im vorherigen Abschnitt beschriebenen Besteuerungs- und Subventionierungsinstrumente eingesetzt werden. Daher muss die Reformbewegung eine Medienpolitik forcieren, welche die Entstehung eines gemeinnützigen, nichtgewerblichen und vom Staat unabhängigen Journalismus vorantreibt (ebd.).

Konkret bedeutet das für die deutsche Medienlandschaft: Der öffentlich-rechtliche Rundfunk sollte als eine zentrale Säule des neuen Mediensystems mittels demokratischer Reformen gestärkt und erweitert werden. Gleichzeitig muss ein neuer, nichtkommerzieller Mediensektor entstehen, der sich insbesondere der Journalismus- und Nachrichtenproduktion widmet. James Curran (2002, 239-241) schlägt vor, diesen Sektor in verschiedene Sphären zu unterteilen, in denen unterschiedliche Gruppierungen der Zivilgesellschaft (insbesondere außerhalb der privilegierten Schichten) eigene Nachrichtenmedien unterhalten können. Das erlaube ihnen, vorherrschende ideologische Annahmen in Frage zu stellen, gemeinsame Interessen zu identifizieren und alternative Sichtweisen auszuloten. Curran (ebd., 239) argumentiert des Weiteren, die Fokussierung von Gruppeninteressen dürfe nicht dem Allgemeininteresse schaden oder gesellschaftliche Konfliktlinien vertiefen: »Mutualism should coexist with freedom and equality as general societal objectives.« Er schlägt daher den Aufbau eines zweigleisigen Mediensystems vor, das einerseits in der Lage sein soll, marginalisierten Gruppen und deren Anliegen, Werten und Identitäten Macht zu verleihen, indem es diesen ermöglicht, ihre eigenen, auf vielfältige Weise organisierten und ideologisch ausgerichteten Nachrichtenmedien zu 
betreiben (ebd., 239-240). Neben diesen Medien der Zivilgesellschaft könnte auch die traditionelle Presse eine Sphäre dieses Teilsystems als privatwirtschaftlich organisierter Nachrichtensektor einnehmen. Anderseits soll das Kernelement der Medien, hier repräsentiert durch den öffentlich-rechtlichen Rundfunk, die Gesellschaft durch die Vermittlung gemeinsamer kultureller Erfahrungen, Werte und Erinnerungen sowie durch die Formierung allgemeiner politischer Debatten zusammenhalten (ebd.).

Auch wäre es denkbar, eine professionell-journalistische Sphäre des Mediensystems im Sinne der von Marie Luise Kiefer (2011) vorgeschlagenen Selbstverwaltung des Journalismus als Commons zu organisieren. Demnach würde er als eine selbstorganisierte Profession institutionalisiert und öffentlich finanziert (ebd., 13-14). Laut Kiefer bedürfe es neben gesellschaftlichen auch »wissenschaftliche, politische und gesetzgeberische Initiativen «, um für dieses Ressourcensystem den relevanten Personenkreis und Regelrahmen sowie Kontroll-, Sanktionsund Konfliktlösungsmechanismen zu etablieren (ebd.). Sie argumentiert, dass die Bildung einer Commons nicht frei von Risiko sei; allerdings spreche vieles dafür, dass professionelle Journalist*innen ihre selbstorganisierte Commons "gemäß den via Ausbildung vermittelten professionellen Regeln und Verpflichtungen pflegen « würden, »wobei die gewonnene Reputation des Berufstandes die quasi-freiwillige Kooperation zusätzlich abstützen dürfte« (ebd., 14). Ihr zufolge könnte dieser Ansatz der Selbstorganisation der einzige Ausweg aus der gegenwärtigen Krise sein, der dem Journalismus die nötige wirtschaftliche Unabhängigkeit und Staatsferne ermöglicht.

\section{$5 \quad$ Zusammenfassung}

Zunächst wurde gezeigt, dass die Krise des Journalismus in Deutschland auf die Institutionen der kapitalistischen Medienindustrie zurückzuführen ist. Daher sind umfangreiche strukturelle Reformen, die in einer Demokratisierung, Neuordnung und Erweiterung des Mediensektors münden, erforderlich. Journalismus und Nachrichtenmedien müssen von den Zwängen des Marktes und der konzernkapitalistischen Institutionen befreit und unter öffentliche Kontrolle gestellt werden. Um das zu erreichen, wurden Visionen und Strategien für Medienreformen sowie ein Katalog konkreter Reformmaßnahmen vorgestellt wie auch, darauf aufbauend, die Konturen eines neuen Mediensystems gezeichnet, das neben 
einer Stärkung und Demokratisierung des Journalismus mehr gesellschaftliche Beteiligung an der Nachrichtenproduktion ermöglichen soll. Medienreform ist ein Prozess, der alle Gesellschaftsgruppen einbeziehen muss. Das kann nur durch die Schaffung einer breitangelegten Bewegung, die von Akteur*innen der Zivilgesellschaft, Wissenschaft, und Regulierungsbehörden getragen wird, passieren. Diese Gruppierungen müssen sich auf lokaler, regionaler und länderübergreifender Ebene vernetzen und ein Programm für eine Medienreform ausarbeiten, das parallel auf die öffentliche Agenda gesetzt werden sollte. Denn eine Medienreform benötigt öffentliche Zustimmung und Partizipation. Nur so können die Gesetzgeber*innen dazu verpflichtet werden, Veränderungen auf politischer Ebene einzuleiten. Die hier vorgestellten Reformen, die überwiegend von einer britisch-amerikanischen Forschungstradition inspiriert sind (Curran 2002; Fenton et al. 2020; Herman 1999; McChesney 1997; 2005; 2008; McChesney et al. 2005), verweisen auf eine mögliche Route, die sicherlich noch einer Verfeinerung bedarf. Denn letztendlich müssen gesellschaftliche Transformationen von der Zivilgesellschaft angestoßen werden. Auch sollten Maßnahmen für umfassende Restrukturierungen des Internet-Sektors, der für die Nachrichtenselektion und -verbreitung zunehmend an Bedeutung gewinnt, Einzug in das Programm der Bewegung halten. In jedem Fall müssen weitere Reformprogramme von Wissenschaft und Zivilgesellschaft erörtert und möglichst bald umgesetzt werden.

\section{Literatur}

Alexander, Jeffrey C. 1981. The Mass News Media in Systemic, Historical, and Comparative Perspective. In: Mass Media and Social Change, herausgegeben von Elihu Katz und Tamás Szecskö, 17-51. London: Sage.

Bakan, Joel. 2005. The Corporation: The Pathological Pursuit of Profit and Power. Überarbeitete und erweiterte Auflage. London: Constable.

Barthel, Michael. 2018. 5 facts about the state of the news media in 2017. http:// www.pewresearch.org/fact-tank/2018/08/21/5-facts-about-the-state-of-thenews-media-in-2017/. Zugegriffen: 9. September 2020.

Becker, Jörg, Frank Götz, und Ulrich Meyerholt. 2007. Der öffentlich-rechtliche Rundfunk zwischen Wettbewerb und Kultur: Gutachten zur Situation und Zukunft der Medien, des Medienrechts und der Medienpolitik in Deutschland und der EU, insbesondere des Öffentlich-rechtlichen Rundfunks. Berlin: Fraktion Die Linke im Deutschen Bundestag.

Beiler, Markus, und Uwe Krüger. 2018. Mehr Mehrwert durch Konstruktiven Journalismus? Idee des Konzepts und Implikationen zur Steigerung des Public Va- 
lues von Medien. In: Der öffentliche (Mehr-)Wert von Medien, herausgegeben von Nicole Gonser, 167-191. Wiesbaden: Springer VS.

Betz, Jürgen. 2015. Der Auftrag des öffentlich-rechtlichen Rundfunks: Anmerkungen zu einem Gutachten. Media Perspektiven 2: 58-65.

Curran, James. 2002. Media and Power. London: Routledge.

Daschmann, Gregor. 2009. Qualität von Fernsehnachrichten: Dimensionen und Befunde. Media Perspektiven 5: 257-266.

Davies, Nick. 2009. Flat Earth News. London: Vintage.

Davies, Nick. 2008. Nick Davies: »Churnalism has taken the place of what we should be doing: Telling the truth«. PressGazette vom 4. Februar, https://www. pressgazette.co.uk/nick-davies-churnalism-has-taken-the-place-of-what-weshould-be-doing-telling-the-truth-40117/. Zugegriffen: 8. September 2020.

Deutscher Bundestag. 2009. Die Staatsferne der Aufsichtsgremien öffentlich-rechtlicher Rundfunkanstalten: Sachstand und Reformansätze in vergleichender Perspektive. WD 10 - 3000 - 044/09, Wissenschaftliche Dienste des Deutschen Bundestages.

Donsbach, Wolfgang, und Katrin Büttner. 2005. Boulevardisierungstrend in deutschen Fernsehnachrichten: Darstellungsmerkmale der Politikberichterstattung vor den Bundestagswahlen 1983, 1990 und 1998. Publizistik 50 (1): 21-38.

Eichler, Boris. 2013. Der graue Block: Der Rundfunk in Deutschland hat staatsfern zu sein. Doch die vermeintlich unabhängigen Vertreter in den Rundfunkräten werden oft von staatlich finanzierten Institutionen entsandt. Liberal 4: 84-87.

Fenton, Natalie, Des Freedman, Justin Schlosberg, und Lina Dencik. 2020. The Media Manifesto. E-Book. Cambridge: Polity Press.

Ferschli, Benjamin, Grabner, Daniel, und Hendrik Theine. 2019. Zur Politischen Ökonomie der Medien in Deutschland: Eine Analyse der Konzentrationstendenzen und Besitzverhältnisse. ISW Report Nr. 118. München: Institut für sozial-ökologische Wirtschaftsforschung.

Heinrich, Jürgen. 2002. Medienökonomie. Band 2: Hörfunk und Fernsehen. 2. Auflage. Wiesbaden: Westdeutscher Verlag.

Herman, Edward S. 1999. The Myth of the Liberal Media: An Edward Herman Reader. New York: Peter Lang.

Herman, Edward S. 1995 Triumph of the Market: Essays on Economics, Politics and the Media. Boston: South End Press.

KEK. 2018. Sicherung der Meinungsvielfalt im digitalen Zeitalter: Bericht der Kommission zur Ermittlung der Konzentration im Medienbereich (KEK) über die Entwicklung der Konzentration und über Maßnahmen zur Sicherung der Meinungsvielfalt im privaten Rundfunk. Schriftenreihe der Landesmediananstalten 52. Berlin: Die Medienanstalten.

Kiefer, Marie Luise. 2011. Die schwierige Finanzierung des Journalismus. Medien \& Kommunikationswissenschaft 59 (1): 5-22.

Kiefer, Marie Luise. 2001. Medienökonomik: Einführung in eine ökonomische Theorie der Medien. München: Oldenbourg.

Klöckner, Marcus B. 2019. Sabotierte Wirklichkeit: Oder: Wenn Journalismus zur Glaubenslehre wird. Frankfurt am Main: Westend. 
Knoche, Manfred. 2013. Krisenhafte kapitalistische Produktionsweise als Triebkraft für Restrukturierungen und Transformationen (in) der Medienindustrie: Erklärende Theorieelemente einer Kritik der politischen Ökonomie der Medien. In: Langfristiger Wandel von Medienstrukturen. Theorie, Methoden, Befunde, herausgegeben von Wolfgang Seufert und Felix Sattelberger, 87-111. Baden-Baden: Nomos.

Leidinger, Christiane. 2003. Medien - Herrschaft - Globalisierung: Folgenabschätzung zu Medieninhalten im Zuge transnationaler Konzentrationsprozesse. Münster: Westfälisches Dampfboot.

Lobings, Frank, und Christoph Neuberger. 2018. Meinungsmacht im Internet und die Digitalstrategien von Medienunternehmen: Neue Machtverhältnisse trotz expandierender Internet-Geschäfte der traditionellen Massenmedien-Konzerne. Leipzig: Vistas.

McChesney, Robert W., Newman, Russell, und Ben Scott. 2005. The Future of Media: Resistance and Reform in the 21st Century. New York: Seven Stories Press.

McChesney, Robert W. 2008. The Political Economy of Media: Enduring Issues, Emerging Dilemmas. New York: Monthly Review Press.

McChesney, Robert W. 2005. The emerging struggle for a free press. In: The Future of Media: Resistance and Reform in the 21st Century, herausgegeben von Robert W. McChesney, Russell Newman und Ben Scott, 9-20. New York: Seven Stories.

McChesney, Robert W. (1997) Corporate Media and the Threat to Democracy. New York: Seven Stories.

Röper, Horst. 2020. Daten zur Konzentration der Tagespresse im I. Quartal 2020. Tageszeitungen 2020: Schrumpfender Markt und sinkende Vielfalt. Media Perspektiven 6: 331-352.

Tröger, Mandy. 2020. Eine Kritik der Ideologie der »freien Presse« zur Wendezeit 1989/1990. In: Ideologie, Kritik, Öffentlichkeit - Verhandlungen des Netzwerks Kritische Kommunikationswissenschaft, herausgegeben von Uwe Krüger und Sebastian Sevignani, 115-135. Frankfurt am Main: Westend.

Weiß, Hans-Jürgen. 2007. Private Fernsehvollprogramme 1998-2007. In: ALM Programmbericht 2007, herausgegeben von Arbeitsgemeinschaft der Landesmedienanstalten in der Bundesrepublik Deutschland, 37-66. Berlin: Vistas.

Wolf, Armin. 2019. Zwischen Propaganda und Paywall: Journalismus in der Krise. Blätter für deutsche und internationale Politik, Januar. https://www.blaetter.de/ ausgabe/2019/januar/zwischen-propaganda-und-paywall-journalismus-inder-krise. Zugegriffen: 8. September 2020.

Zollmann, Florian. 2017. Media, Propaganda and the Politics of Intervention. New York: Peter Lang.

Zollmann, Florian. 2009. Is it Either Or? Professional Ideology vs. Corporate-Media Constraints. Westminster Papers in Communication and Culture 6 (2): 97-118.

\section{Open Access}

Dieser Beitrag erscheint unter der Creative-Commons-Lizenz CC BY-ND 3.0 DE: https://creativecommons.org/licenses/by-nd/3.0/de/. 